\title{
Lower bounds for partial matchings in regular bipartite graphs and applications to the monomer-dimer entropy
}

\author{
Shmuel Friedland \\ Department of Mathematics, \\ Statistics and Computer Science \\ University of Illinois at Chicago \\ Chicago, Illinois 60607-7045 \\ friedlan@uic.edu
}

\author{
Leonid Gurvits \\ Los Alamos \\ National Laboratories \\ Los Alamos \\ NM 87545 \\ gurvits@lanl.gov
}

April 10, 2007

\begin{abstract}
We derive here the Friedland-Tverberg inequality for positive hyperbolic polynomials. This inequality is applied to give lower bounds for the number of matchings in $r$-regular bipartite graphs. It is shown that some of these bounds are asymptotically sharp. We improve the known lower bound for the three dimensional monomer-dimer entropy.
\end{abstract}

2000 Mathematics Subject Classification: 05A15, 05A16, 05C70, 05C80, 82B20

Keywords and phrases: Positive hyperbolic polynomials, Friedland-Tverberg inequality, lower bounds for sum of all subpermanents of doubly stochastic matrices of fixed order, lower bounds for matchings, asymptotic lower matching conjecture, monomerdimer partitions and entropies.

\section{Introduction}

The aim of this paper is to explore the connections between the problem of counting the number of partial matchings in bipartite graphs and positive hyperbolic polynomials. Let $G:=\left(V_{1} \cup V_{2}, E\right)$, where $E \subset V_{1} \times V_{2}$ and $n=\# V_{1}=\# V_{2}$. (We allow graphs with multiple edges.) We want to compute or estimate the number of $m$-matchings in $G$, i.e. the number of subsets $M$ of edges $E$, where $\# M=m$, and no two edges in $M$ have a common vertex.

Let $\mathbb{Z}_{+}$be the set of nonnegative integers. Assume that $A(G)=\left[a_{i j}\right]_{i, j=1}^{n} \in \mathbb{Z}_{+}^{n \times n}$ is the incidence matrix of the bipartite graph $G$, i.e. $a_{i j}$ is the number of edges connecting $i \in V_{1}, j \in V_{2}$. Then the number of $m$-matchings in $G$ is equal to $\operatorname{perm}_{m} A(G)$, where $\operatorname{perm}_{m} A$ is the sum of permanents of all $m \times m$ submatrices of $A \in \mathbb{R}^{n \times n}$. For $m=n$, perm $A(G)$, the permanent of $A(G)$ is the number of perfect matchings in $G$.

We now describe our main results for $\operatorname{perm}_{m} A$, where $A$ is doubly stochastic, and their applications to lower bounds on partial matchings in bipartite graphs. Recall that the minimum of the permanent of $n \times n$ doubly stochastic matrices, denoted by $\Omega_{n}$, is achieved only for the flat matrix $J_{n}$, whose all entries equal to $\frac{1}{n}$. Thus perm $B \geq \operatorname{perm} J_{n}=\frac{n !}{n^{n}}$ for any $B \in \Omega_{n}$ and this inequality was conjectured by van der Waerden [22]. This conjecture was independently proved by Egorichev [3] and Falikman [5]. We call the above inequality Egorichev-Falikman-van der Waerden (EFW) inequality. The asymptotic behavior of EFW inequality is captured by the inequality perm $B \geq e^{-n}$ for any $B \in \Omega_{n}$. This inequality was shown by the first name author [6] two years before [3, 5]. Let $\Gamma(n, r)$ be the set of all $r$-regular bipartite graphs $G$ on $2 n$ vertices, (multiple edges are allowed). For $G \in \Gamma(n, r)$ the matrix $B:=\frac{1}{r} A(G)$ is doubly stochastic. Hence the number of perfect matchings in 
$G$ is at least $\left(\frac{r}{e}\right)^{n}$. Thus for $r \geq 3$, the number of perfect matchings in $r$-regular bipartite graphs grows exponentially, which proves a conjecture by Erdös-Rényi [4]. Schrijver [18] improved the EFW inequality for $r$-regular bipartite graphs by showing that $\operatorname{perm}_{m} A(G) \geq$ $\left(\frac{(r-1)^{r-1}}{r^{r-2}}\right)^{n}$ for any $G \in \Gamma(n, r)$. Schrijver's inequality is asymptotically sharp. Recently, the second name author [14] improved Schrijver's inequality. Moreover, the proof in [14] is significantly simpler and transparent. One of the main tools in the proof in [14] is the use of the classical theory of hyperbolic polynomials.

It was shown by the first named author that $\operatorname{perm}_{m} A \geq \operatorname{perm}_{m} J_{n}$ for any $A \in \Omega_{n}$, and for $m \in[2, n]$ equality holds only if and only if $A=J_{n}[7]$. (perm $1 A=n$ for each $A \in \Omega_{n}$.) This inequality was conjectured by Tverberg [21], and it is called here the Friedland-Tverberg (FT) inequality. FT inequality gives a lower bound on the number of partial matchings in any $G \in \Gamma(n, r)$.

We derive here the Schrijver type inequalities for $m$ matchings in $r$-regular bipartite graphs on $2 n$ vertices. This is done using the results and techniques of [14]. In particular we give a generalized versions of FT inequality to positive homogeneous hyperbolic polynomials, which are of independent interest.

These inequalities yield new lower bounds for the $d$-dimensional monomer-dimer entropy of dimer density $h_{d}(p), p \in[0,1]$ in the lattice $\mathbb{Z}^{d}$. In particular we obtain the best known lower bound for the three dimensional monomer dimer entropy $h_{3}$, which combined with the known upper bound in [11] gives the tight result $h_{3} \in[.7845, .7863]$.

We now list briefly the contents of this paper. In $\S 2$ we discuss briefly the notion of positive hyperbolic polynomials and examples used in this paper. In $\S 3$ we bring the generalized version of FT inequality for positive hyperbolic polynomials. In $\S 4$ we give an analog of the Schrijver-Gurvits inequality to $\operatorname{perm}_{m} B$, where $B$ is a doubly stochastic matrix with at most $r$ nonzero entries in each column. In $\S 5$ we discuss the asymptotic lower matching conjecture (ALMC) and the asymptotic lower $r$-permanent matching conjecture (ALPMC), which is a generalization of ALMC. We show that the main result in $\S 4$ proves the ALMC and ALPMC for a countable values of densities for each $r \geq 2$. In the last section we state the asymptotic upper matching conjectures (AUMC). We illustrate the relations of ALMC and AUMC to the monomer-dimer entropy in statistical mechanics by plotting the corresponding graphs for the dimensions $d=2,3$. We thank Uri Peled for supplying us with the Figures 1 and 2.

\section{Positive hyperbolic polynomials}

\section{Definitions and Notations}

1. A vector $\mathbf{x}:=\left(x_{1}, \ldots, x_{n}\right)^{\top} \in \mathbb{R}^{n}$ is called positive or nonnegative, and denoted by $\mathbf{x}>\mathbf{0}:=(0, \ldots, 0)^{\top}$ or $\mathbf{x} \geq \mathbf{0}$ if $x_{i}>0$ or $x_{i} \geq 0$ for $i=1, \ldots, n$ respectively. A nonnegative vector $\mathbf{x} \neq \mathbf{0}$ is denoted by $\mathbf{x} \geqq \mathbf{0}$. $\mathbf{y} \geq \mathbf{x} \Longleftrightarrow \mathbf{y}-\mathbf{x} \geq \mathbf{0}$. The cone of all nonnegative vectors in $\mathbb{R}^{n}$ is denoted by $\mathbb{R}_{+}^{n}$.

2. A polynomial $p=p(\mathbf{x})=p\left(x_{1}, \ldots, x_{n}\right): \mathbb{R}^{n} \rightarrow \mathbb{R}$ is called positive hyperbolic if the following conditions hold:

- $p$ is a homogeneous polynomial of degree $m \geq 0$.

- $p(\mathbf{x})>0$ for all $\mathbf{x}>0$.

- $\phi(t):=p(\mathbf{x}+t \mathbf{u})$, for $t \in \mathbb{R}$, has $m$-real $t$-roots for each $\mathbf{u}>\mathbf{0}$ and each $\mathbf{x}$.

3. Let $p: \mathbb{R}^{n} \rightarrow \mathbb{R}$ be a positive hyperbolic polynomial of degree $m$. For each integer $i \in[0, n]$ the $i$-th degree of $p$ is the integer $r_{i} \in[0, m]$ such that

$$
\frac{\partial^{r_{i}} p}{\partial x_{i}^{r_{i}}}\left(x_{1}, \ldots, x_{i-1}, 0, x_{i+1}, \ldots, x_{n}\right) \not \equiv 0, \text { and } \frac{\partial^{r_{i}+1} p}{\partial x_{i}^{r_{i}+1}}\left(x_{1}, \ldots, x_{i-1}, 0, x_{i+1}, \ldots, x_{n}\right) \equiv 0 .
$$


Let $\operatorname{deg}_{i} p:=r_{i}$ for $i=1, \ldots, n$.

4. Let $\mathbf{e}_{i}:=\left(\delta_{i 1}, \ldots, \delta_{i n}\right)^{\top} \in \mathbb{R}^{n}, i=1, \ldots, n$ be the standard basis in $\mathbb{R}^{n}$.

5. Let $1:=(1, \ldots, 1)^{\top} \in \mathbb{R}^{n}$ and denote by $J_{n} \in \mathbb{R}^{n \times n}$ the $n \times n$ matrix whose all entries are equal to $\frac{1}{n}$.

We refer to $[8,13,14]$ for properties of positive hyperbolic polynomials used here.

\section{Examples of positive hyperbolic polynomials}

1. Let $A=\left(a_{i j}\right)_{i=j=1}^{m, n} \in \mathbb{R}^{m \times n}$ be a nonnegative matrix, denoted by $A \geq \mathbf{0}$, where each row of $A$ is nonzero. Fix a positive integer $k \in[1, m]$. Then

$$
p_{k, A}(\mathbf{x}):=\sum_{1 \leq i_{1}<\ldots i_{k} \leq m} \prod_{j=1}^{k}(A \mathbf{x})_{i_{j}}, \mathbf{x} \in \mathbb{R}^{n},
$$

is positive hyperbolic of degree $k$ in $n$ variables.

2. Let $A_{1}, \ldots, A_{n} \in \mathbb{C}^{m \times m}$ hermitian, nonnegative definite matrices such that $A_{1}+\ldots+$ $A_{n}$ is a positive definite matrix. Let $p(\mathbf{x})=\operatorname{det} \sum_{i=1}^{n} x_{i} A_{i}$. Then $p(\mathbf{x})$ is positive hyperbolic.

\section{Proof.}

1. First note that $p_{k, A}(\mathbf{x})>0$ for $\mathbf{x}>\mathbf{0}$. The hyperbolicity of $p_{m, A}$ and $p_{1, A}$ is obvious. Assume that $k \in(1, m)$. Let $\mathbf{z}=\left(z_{1}, \ldots, z_{n+m-k}\right)^{\top} \in \mathbb{R}^{n+m-k}$ and define $P(\mathbf{z}):=$ $\prod_{i=1}^{m}\left(\sum_{j=1}^{n} a_{i j} z_{j}+\sum_{j=n+1}^{n+m-k} z_{j}\right)$. Then

$$
p_{k, A}(\mathbf{x})=\left(\begin{array}{c}
m \\
k
\end{array}\right)^{-1} \frac{\partial^{m-k} P}{\partial z_{n+1} \ldots \partial z_{n+m-k}}\left(\left(x_{1}, \ldots, x_{n}, 0, \ldots, 0\right)\right) .
$$

Hence by [8, Lemma 2.1] $p_{k, A}$ positive hyperbolic.

2. This is a standard example and the proof is straightforward.

Let $p(\mathbf{x}): \mathbb{R}^{n} \rightarrow \mathbb{R}$ be a positive hyperbolic polynomial of degree $m \geq 1$. As in [14] define

$$
\text { Cap } p:=\inf _{\mathbf{x}>\mathbf{0}, x_{1} \ldots x_{n}=1} p(\mathbf{x})=\inf _{\mathbf{x}>\mathbf{0}} \frac{p(\mathbf{x})}{\left(x_{1} \ldots x_{n}\right)^{\frac{m}{n}}} .
$$

It is possible that Cap $p=0$. For example let $p=x_{1}^{m_{1}} \ldots x_{n}^{m_{n}}$ where $m_{1}, \ldots, m_{n}$ are nonnegative integer whose sum is $m$ and $\left(m_{1}, \ldots, m_{n}\right) \neq k \mathbf{1}$.

Proposition 2.1 Let $A \in \mathbb{R}^{n \times n}$ be a doubly stochastic matrix. Let $p_{k, A}, k \in[1, n]$ be positive hyperbolic defined as part 1 of the above example. Then Cap $p_{k, A}=\left(\begin{array}{l}n \\ k\end{array}\right)$. Let $B \in \mathbb{R}^{n}$ be a matrix with positive entries. Then there exists two positive definite diagonal matrices $D_{1}, D_{2}$, unique up to $t D_{1}, t^{-1} D_{2}, t>0$, such that $A:=D_{1} B D_{2}$ is a doubly stochastic matrix [20]. Let $p_{n, B}$ be defined as above. Then Cap $p_{n, B}=\frac{1}{\operatorname{det} D_{1} D_{2}}$.

Proof. Consider first $p_{n, A}$. Since $A$ is row stochastic $p_{n, A}(\mathbf{1})=1$. Hence Cap $p_{n, A} \leq 1$. Let $\mathbf{u}=\left(u_{1}, \ldots, u_{n}\right)^{\top} \gtreqless \mathbf{0}$ be a probability vector. Then for any $\mathbf{x}=\left(x_{1}, \ldots, x_{n}\right)>\mathbf{0}$ the generalized arithmetic-geometric inequality states $\mathbf{u}^{\top} \mathbf{x} \geq \prod_{i=1}^{n} x_{i}^{u_{i}}$. Use this inequality for each $(A \mathbf{x})_{i}$. The assumption that $A$ is doubly stochastic yields that $p_{n, A} \geq x_{1} \ldots x_{n} \Rightarrow$ Cap $p_{n, A} \geq 1$. Hence Cap $p_{n, A}=1$. 
Let $k \in[1, n)$. Then $p_{k, A}(\mathbf{1})=\left(\begin{array}{l}n \\ k\end{array}\right)$. Hence Hence Cap $p_{k, A} \leq\left(\begin{array}{l}n \\ k\end{array}\right)$. Apply the arithmeticgeometric inequality to $\frac{p_{k, A}}{\left(\begin{array}{c}n \\ k\end{array}\right)}$ to deduce that $p_{k, A} \geq\left(\begin{array}{l}n \\ k\end{array}\right) p_{n, A}^{\frac{m}{n}}$. Hence Cap $p_{k, A} \geq\left(\begin{array}{l}n \\ k\end{array}\right)$.

It is straightforward to show that $\frac{p_{n, B}(\mathbf{x})}{x_{1} \ldots x_{n}}=\frac{p_{n, A}(\mathbf{y})}{\operatorname{det}\left(D_{1} D_{2}\right) y_{1} \ldots y_{n}}$, where $\mathbf{y}=D_{2}^{-1} \mathbf{x}$. Hence Cap $p_{n, B}=\frac{1}{\operatorname{det} D_{1} D_{2}}$.

The following result is taken from [14].

Lemma 2.2 Let $k \geq 1$ be an integer, $\mathbf{u}:=\left(u_{1}, \ldots, u_{k}\right)^{\top}>\mathbf{0}, \mathbf{v}:=\left(v_{1}, \ldots, v_{k}\right)^{\top}>\mathbf{0}$ and define $f(t):=\prod_{i=1}^{k}\left(u_{i} t+v_{i}\right)$. Let $K(f):=\inf _{t>0} \frac{f(t)}{t}$. Then $f^{\prime}(0)=K$ for $k=1$ and $f^{\prime}(0) \geq\left(\frac{k-1}{k}\right)^{k-1} K$ for $k \geq 2$. For $k \geq 2$ equality holds if and only if $\frac{v_{1}}{u_{1}}=\ldots=\frac{v_{k}}{u_{k}}$.

The following proposition follows straightforward from [8, Lemma 2.1, part 3].

Proposition 2.3 Let $p: \mathbb{R}^{n} \rightarrow \mathbb{R}$ be a positive hyperbolic polynomial of degree $m$. Let $i \in[1, n]$ be an integer. Then

1. $\operatorname{deg}_{i} p=0 \Longleftrightarrow p(\mathbf{x})=\left(p\left(x_{1}, \ldots, x_{i-1}, 0, x_{i+1}, \ldots, x_{n}\right)\right)$.

2. For each integer $j \in\left[0, \operatorname{deg}_{i} p\right] \frac{\partial^{j} p}{\partial x_{i}^{j}}\left(x_{1}, \ldots, x_{i-1}, 0, x_{i+1}, \ldots, x_{n}\right)$ is a positive hyperbolic polynomial of degree $m-j$.

3. For each integer $j \in[1, n], j \neq i$,

$$
\operatorname{deg}_{j} \frac{\partial p}{\partial x_{i}}\left(x_{1}, \ldots, x_{i-1}, 0, x_{i+1}, \ldots, x_{n}\right) \leq \min \left(\operatorname{deg}_{j} p, n-1\right) .
$$

The following result is crucial for the proof of a generalized Friedland-Tverberg inequality and is due essentially to the second author in [14].

Lemma 2.4 Let $p: \mathbb{R}^{n} \rightarrow \mathbb{R}$ be a positive hyperbolic polynomial of degree $m \geq 1$. Assume that Cap $p>0$. Then $\operatorname{deg}_{i} p \geq 1$ for $i=1, \ldots, n$. For $m=n \geq 2$

$$
\text { Cap } \frac{\partial p}{\partial x_{i}}\left(x_{1}, \ldots, x_{i-1}, 0, x_{i+1}, \ldots, x_{n}\right) \geq\left(\frac{\operatorname{deg}_{i} p-1}{\operatorname{deg}_{i} p}\right)^{\operatorname{deg}_{i} p-1} \text { Cap } p \text { for } i=1, \ldots, n,
$$

where $0^{0}=1$.

Proof. It is enough to prove the result for $i=n$. Suppose to the contrary that $p$ does not depend on $x_{n}$. Then let $\mathbf{x}(t)=(1, \ldots, 1, t)^{\top}$ and $t \rightarrow \infty$ in $(2.2)$ to deduce that Cap $p=0$ contrary to our assumption.

Assume that $m=n>1$. Let $k=\operatorname{deg}_{n} p \geq 1$. Let $\mathbf{x}_{0}:=\left(x_{1}, \ldots, x_{n-1}, 0\right)^{\top}, \mathbf{x}_{1}:=$ $\left(x_{1}, \ldots, x_{n-1}\right)^{\top}$. Proposition 2.3 yields that $g\left(\mathbf{x}_{1}\right):=\frac{\partial^{k} p}{\partial x_{i}^{k}}\left(\mathbf{x}_{0}\right)$ is a positive hyperbolic function in $n-1$ variables of degree $m-l$. Hence $g\left(\mathbf{x}_{1}\right)>0$ for $\mathbf{x}_{1}>\mathbf{0}$. Thus for $\mathbf{x}_{1}>\mathbf{0}$

$$
p\left(\mathbf{x}_{0}+t \mathbf{e}_{n}\right)=k ! g\left(\mathbf{x}_{1}\right) t^{l}+\ldots=k ! g\left(\mathbf{x}_{1}\right) \prod_{i=1}^{k}\left(t+\lambda_{i}\left(\mathbf{x}_{1}\right)\right), \quad \lambda_{i}(x)>0, \text { for } i=1, \ldots, k .
$$

The second equality follows from [8, Lemma 2.1, part 2]. Assume in addition that $x_{1} \ldots x_{n-1}=$ 1. Then $\inf _{t>0} \frac{p\left(\mathbf{x}_{0}+t \mathbf{e}_{n}\right)}{t} \geq$ Cap $p$. Apply Lemma 2.2 to the right-hand side of (2.3) to deduce that $\frac{\partial p}{\partial x_{n}}\left(\mathbf{x}_{0}\right) \geq\left(\frac{k-1}{k}\right)^{k-1}$ Cap $p$. Since we assumed that $x_{1} \ldots x_{n-1}=1$ it follows that Cap $\frac{\partial p}{\partial x_{n}}\left(\mathbf{x}_{0}\right) \geq\left(\frac{k-1}{k}\right)^{k-1}$ Cap $p$. 


\section{Friedland-Tverberg inequality}

Theorem 3.1 Let $p: R^{n} \rightarrow \mathbb{R}$ be positive hyperbolic of degree $m \in[1, n]$. Assume that $\operatorname{deg}_{i} p \leq r_{i} \in[1, m]$ for $i=1, \ldots, n$. Rearrange the sequence $r_{1}, \ldots, r_{n}$ in an increasing order $1 \leq r_{1}^{*} \leq r_{2}^{*} \leq \ldots \leq r_{n}^{*}$. Let $k \in[1, n]$ be the smallest integer such that $r_{k}^{*}>m-k$. Then

$$
\begin{array}{r}
\sum_{1 \leq i_{1}<\ldots<i_{m} \leq n} \frac{\partial^{m} p}{\partial x_{i_{1}} \ldots \partial x_{i_{m}}}(\mathbf{0}) \geq \\
\frac{n^{n-m}}{(n-m) !} \frac{(n-k+1) !}{(n-k+1)^{n-k+1}} \prod_{j=1}^{k-1}\left(\frac{r_{j}^{*}+n-m-1}{r_{j}^{*}+n-m}\right)^{r_{j}^{*}+n-m-1} \operatorname{Cap} p .
\end{array}
$$

(Here $0^{0}=1$, and the empty product for $k=1$ is assumed to be 1.) If Cap $>0$ and $r_{i}=m$ for $i=1, \ldots, m$ equality holds if and only if $p=C\left(\frac{x_{1}+\ldots+x_{n}}{n}\right)^{m}$ for each $C>0$.

Proof. Suppose that Cap $p=0$. [8, Lemma 2.1, part 3] yields that the left-hand side of (3.1) is nonnegative and the theorem holds in this case.

Clearly, it is enough to assume the case Cap $p=1$. The case $m=n$ is essentially proven in [14] and we repeat its proof for the convenience of the reader. Permute the coordinates of $x_{1}, \ldots, x_{n}$ such that $\operatorname{deg}_{n} p=\min _{i \in[1, n]} \operatorname{deg}_{i} p \leq r_{1}^{*}$. Assume that $\operatorname{deg}_{n} p=l$. Then Lemma 2.4 yields that $r\left(\left(x_{1}, \ldots, x_{n-1}\right)\right):=\frac{\partial p}{\partial x_{n}}\left(\left(x_{1}, \ldots, x_{n-1}, 0\right)\right)$ is positive hyperbolic of degree $n-1$ and Cap $r \geq\left(\frac{l-1}{l}\right)^{l-1}$ Cap $p$. Since the sequence $\left(\frac{i-1}{i}\right)^{i-1}, i=1, \ldots$, is decreasing to have the lowest possible lower bound we have to assume $l=r_{1}^{*}$. Suppose first that $r_{1}^{*}=n$. Repeating this process $n$ times we get that

$$
\frac{\partial^{n} p}{\partial x_{1} \ldots \partial x_{n}}(\mathbf{0}) \geq \operatorname{Cap} p \prod_{j=2}^{n}\left(\frac{j-1}{j}\right)^{j-1}=\frac{n !}{n^{n}} \operatorname{Cap} p .
$$

This inequality to corresponds to the case $r_{i}^{*}=n$ for $i=1, \ldots, n$. The equality case is discussed in [14].

Let $m \in[1, n-1]$. Put $P(\mathbf{x})=p(\mathbf{x})\left(\frac{1}{n} \sum_{i=1}^{n} x_{i}\right)^{n-m}$. Clearly, $P$ is positive hyperbolic of degree $n$. Since $\frac{1}{n} \sum_{i=1}^{n} x_{i} \geq\left(x_{1} \ldots x_{n}\right)^{\frac{1}{n}}$ for each $\mathbf{x} \geq 0$, it follows that Cap $P \geq \operatorname{Cap} p$. Apply (3.1) to $P$ for $m=n$ to deduce (3.1) in the general case. Since the equality case for $P$ holds if and only $P=\left(\frac{1}{n} \sum_{i=1}^{n} x_{i}\right)^{n}$ it follows that the equality in (3.1) holds if and only if $p=\left(\frac{1}{n} \sum_{i=1}^{n} x_{i}\right)^{m}$.

Let $A \in \mathbb{R}^{n \times n}$ be a doubly stochastic matrix. Apply this theorem to $p_{m, A}$ defined Proposition 2.1 to deduce the Friedland-Tverberg inequality for the sum of all $m \times m$ permanents of $A$ :

Corollary 3.2 Let $A \in \mathbb{R}_{+}^{n \times n}$ be a doubly stochastic matrix. Then $\operatorname{perm}_{m} A \geq\left(\begin{array}{c}n \\ m\end{array}\right)^{2} \frac{m !}{n^{m}}$ for any $m \in[2, n]$. equality holds if and only $A=J_{n}$.

Theorem 3.3 (Gurvits) Let $A \in \mathbb{R}_{+}^{n \times n}$ be a doubly stochastic matrix, such that each column contains at most $r \in[1, n]$ nonzero entries. Then

$$
\operatorname{perm} A \geq \frac{r !}{r^{r}}\left(\frac{r-1}{r}\right)^{(r-1)(n-r)}=\frac{r !}{r^{r}}\left(\frac{r}{r-1}\right)^{r(r-1)}\left(\frac{r-1}{r}\right)^{(r-1) n} .
$$

Proof. Note that for $p(\mathbf{x})=\prod_{i=1}^{n}(A \mathbf{x})_{i}$ we have that $\operatorname{deg}_{i} p=r$ for $i=1, \ldots, n$. Apply (3.1) to this case, i.e. $m=n, r_{j}^{*}=r, j=1, \ldots, n$ and $k=n-r+1$ to deduce the theorem. 


\section{A lower bound for partial matchings in $\Gamma(n, r)$}

The FT inequality yields the following lower bound on $m$ matchings in $\Gamma(n, r)$

$$
\operatorname{perm}_{m}(A(G)) \geq r^{m} \operatorname{perm}_{m} J_{n}=\left(\begin{array}{c}
n \\
m
\end{array}\right)^{2} \frac{m ! r^{m}}{n^{m}}, \quad \text { for all } G \in \Gamma(n, r) .
$$

In order to improve this inequality, as done by Schrijver, one has to use the fact that $A(G)$ has at most $r$ nonzero entries in each column. Unfortunately, Theorem 3.1 does not improve the inequality (4.1) for large values of $n$, where $\frac{m}{n} \approx t \in(0,1)$. This is due to the fact that the proof of Theorem 3.1 for a polynomial $p$ is obtained by considering the polynomial $q=p\left(x_{1}+\ldots+x_{n}\right)^{n-m}$. (Note that $\operatorname{deg}_{i} q=\operatorname{deg}_{i} p+n-m$ for $i=1, \ldots, n$.)

In order to use efficiently the fact $\operatorname{deg}_{i} p_{m, A(G)} \leq r$ for $i=1, \ldots, n$, where $A(G), G \in$ $\Gamma(n, r)$, one needs to consider the product $p_{m, A(G)} q(x)$, where $\operatorname{deg} q=n-m$ and $\operatorname{deg}_{i} q \leq s$ for $i=1, \ldots, n$. This $q$ should be a highly symmetric polynomial, similar to $\left(x_{1}+\ldots+\right.$ $\left.x_{n}\right)^{n-m}$. We find such $q$ by averaging $p_{n-m, A(H)}, H \in \Gamma(n, s)$ with respect to a certain "natural" measure on $\Gamma(n, s)$, [17]. (Note that $q$ may not be hyperbolic, but since it is a convex combination of hyperbolic polynomials, we can use Theorem 3.1.)

Let $S_{N}$ be the permutation group on $\{1, \ldots, N\}$. We now give a map $\tau: S_{n r} \rightarrow \Gamma(n, r)$. Fix $\mu \in S_{n r}$. Let $e_{1}, \ldots, e_{n r}$ be $n r$ edges going from vertices $\{1, \ldots, n\}$ in the group $V_{1}$ to vertices $\{1, \ldots, n\}$ to the group $V_{2}$ as follows. Each $e_{i}$ connects the vertex $\left\lceil\frac{i}{r}\right\rceil$ in group $V_{1}$ to $\left\lceil\frac{\mu(i)}{r}\right\rceil$ in group $V_{2}$ for $i=1, \ldots, r n$. Note that the vertex $i$ in group $V_{1}$ has $r$ edges labeled $r(i-1)+1, \ldots, r i$. It is straightforward to see that each vertex $j$ in the group $V_{2}$ has $r$ different edges connected to it, i.e. the equation $j=\left\lceil\frac{\mu(i)}{r}\right\rceil$ has exactly $r$ integers $\mu^{-1}(\{j(r-1)+1, \ldots, j r\})$. It is not difficult to show that $\tau$ is onto. Let $\# \tau^{-1}(G)$ be the number of preimages of $G \in \Gamma(n, r)$ in $S_{n r}$. Denote by $\nu(n, r)$ the probability measure on $\Gamma(n, r)$ given by $\frac{\# \tau^{-1}(G)}{(r n) !}$. The following lemma is taken from [10] and we bring its proof for completeness.

Lemma 4.1 Let $\nu(n, r)$ be the probability measure on $\Gamma(n, r)$ defined above. Then

$$
E_{\nu(n, r)}\left(\operatorname{perm}_{m} A(G)\right)=\frac{\left(\begin{array}{c}
n \\
m
\end{array}\right)^{2} r^{2 m} m !(r n-m) !}{(r n) !} .
$$

Proof. We adopt the arguments of [18] to our case. First choose subset $\alpha \subset\{1, \ldots, n\}$ of $m$ vertices in the group $V_{1}$. There are $\left(\begin{array}{c}n \\ m\end{array}\right)$ choices for $\alpha$. $\alpha$ induces the set $I=$ $\cup_{i \in \alpha}\{r(i-1)+1, \ldots, i r\}$ of edges of cardinality $r m$. From $I$ choose a set $J=\{1 \leq$ $\left.j_{1}<\ldots<j_{m} \leq n r\right\}$ of $m$ edges, so that $e_{j}, j \in J$ corresponds to the choice of one element in the group $\{r(i-1)+1, \ldots, i r\}$, for each $i \in \alpha$. There are $r^{m}$ of the choices of $J$. Now we want to choose $\mu$ so that $\left\lceil\frac{\mu(j)}{r}\right\rceil, j \in J$ will be a subset of $m$ distinct elements $\beta=\left\{\left\lceil\frac{\mu\left(j_{1}\right)}{r}\right\rceil, \ldots,\left\lceil\frac{\mu\left(j_{m}\right)}{r}\right\rceil\right\} \subset\{1, \ldots, n\}$. There are $\left(\begin{array}{c}n \\ m\end{array}\right)$ such choices of $\beta$. Given $\beta \subset\{1, \ldots, n\}$ we can permute the order of elements in $\beta$ in $m$ ! ways. Altogether we have $m !\left(\begin{array}{c}n \\ m\end{array}\right)$ choices of $\left\lceil\frac{\mu\left(j_{1}\right)}{r}\right\rceil, \ldots,\left\lceil\frac{\mu\left(j_{m}\right)}{r}\right\rceil$. Then $\mu(j) \in\left\{\left\lceil\frac{\mu(j)}{r}\right\rceil(r-1)+1, \ldots,\left\lceil\frac{\mu(j)}{r}\right\rceil r\right\}$ for each $j \in J$. Again there are $r^{m}$ such choices. Thus we chose $\mu$ by determining the image of the elements in $J$ in $\{1, \ldots, n r\}$, which is denoted by $\beta:=\mu(J)$. The rest of the of elements $\{1, \ldots, r n\} \backslash J$ is mapped to $\{1, \ldots, r n\} \backslash \beta$. The number of choices here is $(n r-m)$ !. Multiply all these choices to get the numerator of the right-hand side of (4.2). Divide these number of choices by the number of permutations of $\{1, \ldots, r n\}$ to deduce the lemma.

The case $m=n$ in (4.2) is given in [17]. The proof of the above Lemma yields:

Corollary 4.2 For $\beta \subset\langle n\rangle, \# \beta=m$ and $G \in \Gamma(n, r)$ let $\psi(G, \beta)$ be all m-matching in $G$ that cover the set $\beta \subset V_{2}$. Then

$$
E_{\nu(n, r)}(\psi(G, \beta))=\frac{\left(\begin{array}{c}
n \\
m
\end{array}\right) r^{2 m} m !(r n-m) !}{(r n) !}, \quad m=0, \ldots, n .
$$


Remark 4.3 The probability measure $\nu(n, r)$ on $\Gamma(n, r)$ was used in [19] to get an upper bound: $\min _{G \in \Gamma(n, r)} \operatorname{perm}(A(G)) \leq E_{\nu(n, r)}(\operatorname{perm}(A(G))$. The proof of the lower bound in [18], substantially harder result, had no connection to $\nu(n, r)$.

Quite surprisingly, we use in this paper the measure $\nu(n, s)$ to obtain a lower bound. This combination of the "hyperbolic polynomials approach" and the probabilistic method is the main contribution of our paper.

Theorem 4.4 Let $p: R^{n} \rightarrow \mathbb{R}$ be positive hyperbolic of degree $m \in[1, n)$. Assume that $\operatorname{deg}_{i} p \leq r_{i} \in[1, m]$ for $i=1, \ldots, n$. Rearrange the sequence $r_{1}, \ldots, r_{n}$ in an increasing order $1 \leq r_{1}^{*} \leq r_{2}^{*} \leq \ldots \leq r_{n}^{*}$. Let $s \in \mathbb{N}$. Let $k \in[1, n]$ be the smallest integer such that $r_{k}^{*}+s>n-k$. Then

$$
\begin{array}{r}
\sum_{1 \leq i_{1}<\ldots<i_{m} \leq n} \frac{\partial^{m} p}{\partial x_{i_{1}} \ldots \partial x_{i_{m}}}(\mathbf{0}) \geq \\
\frac{(s n) !}{s^{n-m}(n-m) !((s-1) n+m) !} \frac{(n-k+1) !}{(n-k+1)^{n-k+1}} \prod_{j=1}^{k-1}\left(\frac{r_{j}^{*}+s-1}{r_{j}^{*}+s}\right)^{r_{j}^{*}+s-1} \text { Cap } p .
\end{array}
$$

Proof. Let $q: \mathbb{R}^{n} \rightarrow \mathbb{R}$ be positive hyperbolic of degree $n-m$ with $\operatorname{deg}_{i} q \leq s$ for $i=1, \ldots, n$ and Cap $q=1$. Then $f=p q: \mathbb{R}^{n} \rightarrow \mathbb{R}$ is positive hyperbolic of degree $n$ with Cap $f \geq \operatorname{Cap} p$ and $\operatorname{deg}_{i} f \leq r_{i}+s$ for $i=1, \ldots, n$. Apply Theorem 3.1 to $f$ to deduce

$$
\begin{gathered}
\sum_{1 \leq i_{1}<\ldots<i_{m} \leq n} \frac{\partial^{m} p}{\partial x_{i_{1}} \ldots \partial x_{i_{m}}}(\mathbf{0}) \frac{\partial^{n-m} q}{\partial x_{i_{1}^{\prime}} \ldots \partial x_{i_{n-m}^{\prime}}}(\mathbf{0}) \geq \\
\frac{(n-k+1) !}{(n-k+1)^{n-k+1}} \prod_{j=1}^{k-1}\left(\frac{r_{j}^{*}+s-1}{r_{j}^{*}+s}\right)^{r_{j}^{*}+s-1} \operatorname{Cap} p,
\end{gathered}
$$

where $I^{\prime}:=\left\{1 \leq i_{1}^{\prime}<\ldots<i_{n-m}^{\prime} \leq n\right\}$ and $\left\{i_{1}, \ldots, i_{m}, i_{1}^{\prime}, \ldots, i_{n-m}^{\prime}\right\}=\langle n\rangle$.

Let $A:=A(G), G \in \Gamma(n, s)$ and choose $q=\left(\begin{array}{c}n \\ n-m\end{array}\right)^{-1} p_{n-m, \frac{1}{s} A}(\mathbf{x})$ as in (2.1). Note

$$
\frac{\partial^{n-m} q}{\partial x_{i_{1}^{\prime}} \ldots \partial x_{i_{n-m}^{\prime}}}(\mathbf{0})=\frac{1}{\left(\begin{array}{c}
n \\
n-m
\end{array}\right) s^{n-m}} \psi\left(G, I^{\prime}\right) .
$$

Now take the expected value of the left-hand side of the inequalities (4.5) corresponding to all $G \in \Gamma(n, s)$. Use Corollary 4.2 to deduce that the coefficient of each $\frac{\partial^{m} p}{\partial x_{i_{1}} \ldots \partial x_{i_{m}}}(\mathbf{0})$ is $\frac{s^{n-m}(n-m) !((s-1) n+m) !}{(s n) !}$.

Corollary 4.5 Let $p: R^{n} \rightarrow \mathbb{R}$ be positive hyperbolic of degree $m \in[1, n)$. Assume that $\operatorname{deg}_{i} p \leq r \in[1, m]$ for $i=1, \ldots, n$. Let $s \in \mathbb{N}$ and $k=n-r-s+1 \geq 1$. Then

$$
\begin{array}{r}
\sum_{1 \leq i_{1}<\ldots<i_{m} \leq n} \frac{\partial^{m} p}{\partial x_{i_{1}} \ldots \partial x_{i_{m}}}(\mathbf{0}) \geq \\
\frac{(s n) !}{s^{n-m}(n-m) !((s-1) n+m) !} \frac{(r+s) !}{(r+s)^{r+s}}\left(\frac{r+s-1}{r+s}\right)^{(r+s-1)(n-r-s)} \operatorname{Cap} p .
\end{array}
$$

Theorem 4.6 Let $B \in \mathbb{R}_{+}^{n \times n}$ be a doubly stochastic matrix with at most $r$ nonzero entries in each column. Let $s \in \mathbb{N}$ and $k=n-r-s+1 \geq 1$. Then for each $m \in[1, n)$

$$
\operatorname{perm}_{m} B \geq \frac{(s n) !\left(\begin{array}{c}
n \\
m
\end{array}\right)}{s^{n-m}(n-m) !((s-1) n+m) !} \frac{(r+s) !}{(r+s)^{r+s}}\left(\frac{r+s-1}{r+s}\right)^{(r+s-1)(n-r-s)} .
$$

Proof. Let $p=p_{m, B}(\mathbf{x})$ as defined by (2.1). Then (4.7) follow from Corollary 4.5. 


\section{$5 \quad$ ALMC and ALPMC}

Let $G=(V, E)$ be a general, (not necessary bipartite), graph with the set of vertices $V$ and edges $E$. A matching in $G$ is a subset $M \subseteq E$ such that no two edges in $M$ share a common endpoint. The endpoints of the edges in $M$ are said to be covered by $M$. We can think of each edge $e=(u, v) \in M$ as occupied by a dimer, consisting of two neighboring atoms at $u$ and $v$ forming a bond, and of each vertex not covered by $M$ as a monomer, which is an atom not forming any bond. For this reason a matching in $G$ is also called a monomer-dimer cover of $G$. If there are no monomers, $M$ is said to be a perfect matching. Note that if a perfect matching exists then $\# V$ is even. A matching $M$ with $\# M=k$ is called an $k$-matching. We denote by $\phi_{G}(k)$ be the number of $k$-matchings in $G$ (in particular $\phi_{G}(0)=1$ ), and by $\Phi_{G}(x):=\sum_{k} \phi_{G}(k) x^{k}$ the matching generating polynomial of $G$. It is known that all the roots of matching polynomial are real negative numbers [17]. Assume that $G$ is a bipartite graph $G=(V, E)$, where $V=V_{1} \cup V_{2}$ and $\# V_{1}=\# V_{2}=n$. Then $\phi_{G}(k)=\operatorname{perm}_{k} A(G)$.

The following asymptotic result is shown in [10]. (It follows straightforward from Lemma 4.1.)

Theorem 5.1 Let $\nu(n, r)$ be the probability measure on $\Gamma(n, r)$ defined in $\S 4$. Let $j_{n} \in$ $[1, n], n=1,2, \ldots$ be a sequence of integers with $\lim _{n \rightarrow \infty} \frac{j_{n}}{n}=t \in[0,1]$. Then

$$
\lim _{n \rightarrow \infty} \frac{\log E_{\nu(n, r)}\left(\operatorname{perm}_{j_{n}} A(G)\right)}{2 n}=g h_{r}(t),
$$

where

$$
g h_{r}(t):=\frac{1}{2}\left(t \log r-t \log t-2(1-t) \log (1-t)+(r-t) \log \left(1-\frac{t}{r}\right)\right),
$$

An equivalent form of the following conjecture is stated in [9].

Conjecture 5.2 (The Asymptotic Lower Matching Conjecture)

Let $r \geq 2$ be an integer, $\left\{k_{l}\right\}_{l=1}^{\infty},\left\{n_{l}\right\}_{l=1}^{\infty}$ be two increasing sequences of positive integers such that $k_{l} \leq n_{l}, l=1, \ldots$, and $\lim _{l \rightarrow \infty} \frac{k_{l}}{n_{l}}=t \in[0,1]$. Then

$$
\lim _{l \rightarrow \infty} \frac{\log \min _{G \in \Gamma\left(r, n_{l}\right)} \operatorname{perm}_{k_{l}} A(G)}{2 n_{l}}=g h_{r}(t) .
$$

For $r=1$ this conjecture holds trivially. For $r=2$ this conjecture is proved in [10]. The inequality (4.1) implies that under the conditions of Conjecture 5.2 the following inequality holds, see [11]

$$
\liminf _{l \rightarrow \infty} \frac{\log \min _{G \in \Gamma\left(r, n_{l}\right)} \operatorname{perm}_{k_{l}} A(G)}{2 n_{l}} \geq f h_{r}(t),
$$

where

$$
f h_{r}(t):=\frac{1}{2}(-t \log t-2(1-t) \log (1-t)+t \log r-t) .
$$

Definition 5.3 For $r \in \mathbb{N}$ let $\Omega_{n, r}$ be the set of all $n \times n$ doubly stochastic matrices with at most $r$ nonzero entries in each column.

Note that for $G \in \Gamma(n, r) B:=\frac{1}{r} A(G) \in \Omega_{n, r}$. Hence, the ALMC conjecture follows from the following stronger conjecture:

\section{Conjecture 5.4 (The Asymptotic Lower $r$-Permanent Conjecture )}

Let $r \geq 2$ be an integer, $\left\{k_{l}\right\}_{l=1}^{\infty},\left\{n_{l}\right\}_{l=1}^{\infty}$ be two increasing sequences of positive integers such that $k_{l} \leq n_{l}, l=1, \ldots$, and $\lim _{l \rightarrow \infty} \frac{\overline{\bar{k}}_{l}}{n_{l}}=t \in[0,1]$. Then

$$
\lim _{l \rightarrow \infty} \frac{\log \min _{B \in \Omega_{n_{l}, r}} \operatorname{perm}_{k_{l}} B}{2 n_{l}}=g h_{r}(t)-\frac{t}{2} \log r .
$$


Theorem 5.5 Let $r \geq 2, s \geq 1$ be integers. Let $\left\{k_{l}\right\}_{l=1}^{\infty},\left\{n_{l}\right\}_{l=1}^{\infty}$ be two increasing sequences of positive integers such that $k_{l} \leq n_{l}, l=1, \ldots$, and $\lim _{l \rightarrow \infty} \frac{k_{l}}{n_{l}}=t \in[0,1]$. Assume that $B_{n_{l}} \in \Omega_{n_{l}, r}, l=1,2, \ldots$ Then

$$
\begin{aligned}
& \liminf _{l \rightarrow \infty} \frac{\log \operatorname{perm}_{k_{l}} B_{n_{l}}}{2 n_{l}} \geq \frac{1}{2}(-t \log t-2(1-t) \log (1-t))+ \\
& \frac{1}{2}\left((r+s-1) \log \left(1-\frac{1}{r+s}\right)-(s-1+t) \log \left(1-\frac{1-t}{s}\right)\right) .
\end{aligned}
$$

Hence the Asymptotic Lower $r$-Permanent Conjecture 5.4 and ALMC holds for $t_{s}=\frac{r}{r+s}, s=$ $0,1,2, \ldots$, and $t=0$.

Proof. Apply the inequality (4.7) to $B_{n_{l}}$ for $m=k_{l}$. Take the logarithm of the both sides of this inequality and let $l \rightarrow \infty$. A straightforward calculation for the right-hand side, using the Stirling's formula, yields the inequality (5.7). Assume that $t=t_{s}=\frac{r}{r+s}$. Then $\frac{1-t_{s}}{s}=\frac{1}{r+s}=\frac{t_{s}}{r}$. Then the right-and side of (5.7) is equal to $g h_{r}\left(t_{s}\right)-\frac{t_{s}}{2} \log r$.

We now show that the Asymptotic Lower $r$-Permanent Conjecture 5.4 holds for $t_{s}=\frac{r}{r+s}$, where $s$ is any nonnegative integer, and for $t_{\infty}:=0$. Assume that conditions of Conjecture 5.4. Lemma 4.1 implies that there exists $G_{l} \in \Gamma\left(r, n_{l}\right)$ so that

$$
\operatorname{perm}_{k_{l}} \frac{1}{r} A\left(G_{l}\right) \leq \frac{\left(\begin{array}{c}
n_{l} \\
k_{l}
\end{array}\right)^{2} r^{k_{l}}\left(k_{l}\right) !\left(r n_{l}-k_{l}\right) !}{\left(r n_{l}\right) !} .
$$

Clearly, $B_{n_{l}}:=\frac{1}{r} A\left(G_{l}\right) \in \Omega_{n_{l}, r}$ for each $l$. Use Theorem 5.1 to deduce that for this sequence $B_{n_{l}}, l \in \mathbb{N}$, limsup of the left-hand side of (5.6) is at most $g h_{r}(t)-\frac{t}{2} \log r$.

Let $s \in \mathbb{N}$ and $t_{s}=\frac{r}{r+s}$. (5.7) implies that lim inf of the sequence given by the left-hand side of (5.6) is not less than $g h_{r}\left(t_{s}\right)-\frac{t_{s}}{2} \log r$. Hence (5.6) holds for $t=t_{s}$.

We now discuss the case $s=0$, i.e. $t=t_{0}=1$. Let $B=\left(b_{i j}\right)_{i, j=1}^{n}$ be any $n \times n$ nonnegative matrix. Denote by $G(B)=(V, E)$ the bipartite graph induced by $B$, i.e. the edge $(i, j)$ is in $E$, if and only if $b_{i j}>0$. Then $B$ induces the weighted graph on $G$, where the weight of the edge $(i, j)$ is $b_{i j}$. Let $p_{B}(x)=x^{n}+\sum_{m=1}^{n}(-1)^{m} \operatorname{perm}_{m} B \cdot p_{B}(x)$ is called the matching polynomial of the weighted graph $G$. Heilmann and Lieb showed in [16] that $p_{B}(x)$ has nonnegative roots. (See also [17].) Hence the arithmetic-geometric inequality for the elementary symmetric polynomials of the nonnegative roots of $p_{B}(x)$ yields the inequality $\operatorname{perm}_{m} B \geq\left(\begin{array}{c}n \\ m\end{array}\right)(\text { perm } B)^{\frac{m}{n}}$. (See [23] for the case of $m$-matchings in bipartite graphs.)

Use Theorem 3.3 to deduce that perm $B_{n} \geq \frac{r !}{r^{r}}\left(\frac{r}{r-1}\right)^{r(r-1)}\left(\frac{r-1}{r}\right)^{(r-1) n}$ for any $B \in \Omega_{n, r}$. Apply the above two inequalities for the sequence $B_{n_{l}}$ and $m=k_{l}$ for $l=1,2, \ldots$ to obtain that lim inf of the sequence given by the left-hand side of (5.6) is not less than $g h_{r}(1)-\frac{1}{2} \log r$ for $t=1$. Hence (5.6) for $t=1$.

The case $t=0$ follows from (5.4) for $t=0$. As we noted above the Asymptotic Lower $r$-Permanent Conjecture 5.4 for $t=\tau$ implies the ALMC for $t=\tau$.

Let $C_{n}$ a cycle on $n$ vertices, and let $T_{n, d}=\left(V_{n}, E_{n}\right):=\underbrace{C_{n} \times \ldots \times C_{n}}_{d}, n=3, \ldots$ be a sequence of $d$ dimensional torii. Note that each $T_{n, d}$ is $2 d$ regular graph. It is a classical result that the following limit exists for any $t \in[0,1]$ :

$$
\lim _{n \rightarrow \infty} \frac{\log \phi_{T_{n, d}}\left(j_{n}\right)}{\# V_{n}}=h_{d}(t) \text {, if } \lim _{n \rightarrow \infty} \frac{2 j_{n}}{\# V_{n}}=t \in[0,1] .
$$

$h_{d}(t)$ is the $d$-dimensional monomer-dimer entropy of dimer density $t \in[0,1]$ in the lattice $\mathbb{Z}^{d}[15]$ and [11]. Let $h_{d}:=\max _{t \in[0,1]} h_{d}(t)$. (The quantities $h_{d}$ and $\tilde{h}_{d}:=h_{d}(1)$ are called the $d$-monomer-dimer entropy and the 2-dimer entropy, respectively, in [11]).

Let $d=6$ and $t_{3}:=\frac{6}{9}=\frac{2}{3}$. The validity of ALMC for $t_{3}$ yields that $h_{3}\left(\frac{2}{3}\right) \geq .7845241927$, which implies that $h_{3}=\max _{t \in[0,1]} h_{3}(t) \geq .7845241927$. This improves the lower bound implied by (5.4) $h_{3} \geq .7652789557$ [11]. The computations in [11] yield that $h_{3} \leq .7862023450$. Thus $h_{3} \in[.7845, .7863]$. 


\section{The AUMC and related graphs}

Denote by $\mathbb{R}[x]$ the algebra of polynomials in $x$ with real coefficients, by $0 \in \mathbb{R}[x]$ the zero polynomial, and by $\mathbb{R}_{+}[x] \subset \mathbb{R}[x]$ the subalgebra of polynomials with non-negative coefficients. We partially order $\mathbb{R}[x]$ by writing, for $f, g \in \mathbb{R}[x], g \succeq f$ when $g-f \in \mathbb{R}_{+}[x]$, and $g \succ f$ when $g-f \in \mathbb{R}_{+}[x] \backslash\{0\}$. Clearly, if $g_{1} \succeq f_{1} \succ 0$ and $g_{2} \succeq f_{2} \succ 0$, then $g_{1} g_{2} \succ f_{1} f_{2}$ unless $g_{1}=f_{1}$ and $g_{2}=f_{2}$.

Let $q K_{r, r}$ denote the union of $q$ complete bipartite graphs $K_{r, r}$ having $r$ vertices of each color class. It is straightforward to show that any finite graphs $G, G^{\prime}$ satisfy

$$
\Phi_{G \cup G^{\prime}}(x)=\Phi_{G}(x) \Phi_{G^{\prime}}(x),
$$

and that

$$
\Phi_{K_{r, r}}(x)=\sum_{k=0}^{r}\left(\begin{array}{l}
r \\
k
\end{array}\right)^{2} k ! x^{k} .
$$

The following conjecture is stated in [9]

Conjecture 6.1 (The Upper Matching Conjecture) Let $G$ be a bipartite $r$-regular graph on $2 q r$ vertices where $q, r \geq 2$. Then $\Phi_{G} \preceq \Phi_{q K_{r, r}}$, equality holding only if $G=q K_{r, r}$.

For $k=2$ this conjecture is proved in [10]. The above conjecture implies the following Asymptotic Upper Matching Conjecture [9]. Denote by $K(r)$ be the countably infinite union of $K_{r, r}$. Let $P_{K(r)}(u)$ and $h_{K(r)}(t), t \in(0,1)$ be the pressure and the $t$-matching entropy associated and the with $K(r)$ [12]:

$$
\begin{array}{cc}
P_{K(r)}(u)=\frac{\log \sum_{k=0}^{r}\left(\begin{array}{l}
r \\
k
\end{array}\right)^{2} k ! e^{2 k u}}{2 r}, & u \in \mathbb{R} . \\
h_{K(r)}(t(u))=P_{K(r)}(u)-u t(u), & u \in \mathbb{R}
\end{array}
$$

where

$$
t(u)=P_{K(r)}^{\prime}(u)=\frac{\sum_{k=0}^{r}\left(\begin{array}{l}
r \\
k
\end{array}\right)^{2} k !(2 k) e^{2 k u}}{2 r \sum_{k=0}^{r}\left(\begin{array}{l}
r \\
k
\end{array}\right)^{2} k ! e^{2 k u}}, \quad u \in \mathbb{R} .
$$

\section{Conjecture 6.2 (The Asymptotic Upper Matching Conjecture)}

Let $r \geq 2$ be an integer, $\left\{k_{l}\right\}_{l=1}^{\infty},\left\{n_{l}\right\}_{l=1}^{\infty}$ be two increasing sequences of positive integers such that $k_{l} \leq n_{l}, l=1, \ldots$, and $\lim _{l \rightarrow \infty} \frac{k_{l}}{n_{l}}=t \in[0,1]$. Then Then

$$
\lim _{l \rightarrow \infty} \frac{\log \max _{G \in \Gamma\left(n_{l}, r\right)} \operatorname{perm}_{k_{l}} A(G)}{2 n_{l}}=h_{K(r)}(t) .
$$

For $r=2$ the AUMC is proven in [10]. For $t=1$ and any $r \in \mathbb{N}$ the AUMC follows from the proof of Minc conjecture by Bregman [2]. Some computations performed in [9] support the ALMC and AUMC.

The following plots illustrating the Asymptotic Matching Conjectures for $r=4,6$. Figure 1 shows various bounds and values for the monomer-dimer entropy $h_{2}(p)$ of dimer density $p \in[0,1]$ in the 4-regular 2-dimensional grid. FT is the Friedland-Tverberg lower bound $f h_{4}(p)$ of $(5.5), \mathrm{h} 2$ is the true monomer-dimer entropy equal to $\max _{p \in[0,1]} h_{2}(p)$ (it is known to a precision much greater than the picture resolution). The crosses marked B are Baxter's computed values [1]. (Baxter's computations, based on heuristic arguments, where confirmed by theoretical rigorous computations in [12].) ALMC is the function $g h_{4}(p)$ of $(5.2)$, conjectured to be a lower bound in the Asymptotic Lower Matching Conjecture. AUMC is the monomer-dimer entropy $h_{K}(p)$ of dimer density $t$ in a countable union of $K_{4,4}$, given by (6.3)-(6.5) and conjectured to be an upper bound by the Asymptotic Upper Matching Conjecture. Notice that AUMC goes a little over h2: a countable union of $K_{4,4}$ has a higher monomer-dimer entropy than an infinite planar grid. 


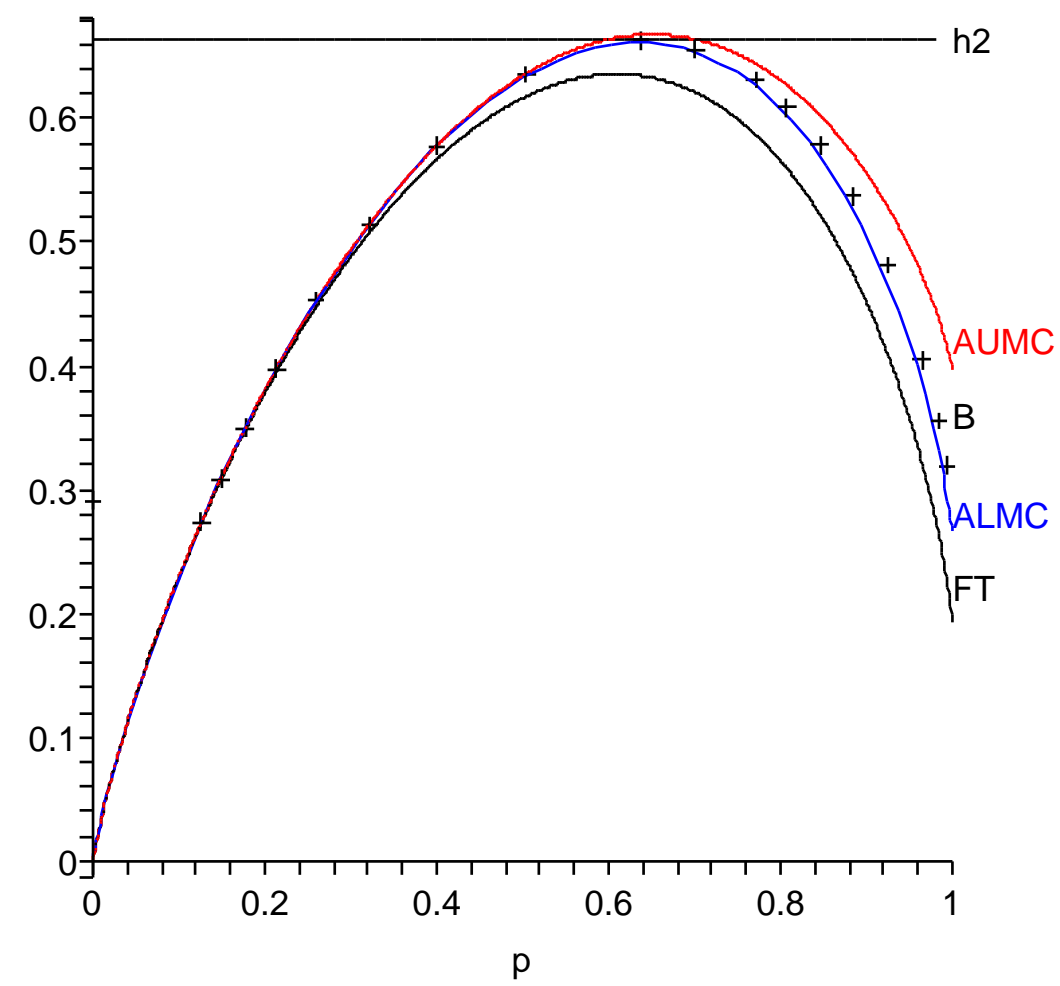

Figure 1: Monomer-dimer tiling of the 2-dimensional grid: entropy as a function of dimer density. FT is the Friedland-Tverberg lower bound, h2 is the true monomer-dimer entropy. $\mathrm{B}$ are Baxter's computed values. ALMC is the Asymptotic Lower Matching Conjecture. AUMC is the entropy of a countable union of $K_{4,4}$, conjectured to be an upper bound by the Asymptotic Upper Matching Conjecture.

Figure 2 shows similarly various bounds and values for the monomer-dimer entropy $h_{3}(p)$ of dimer density $p \in[0,1]$ in the 6 -regular 3 -dimensional grid. FT is the FriedlandTverberg lower bound $f h_{6}(p)$ of (5.5). h3High is the best known upper bounds for the true monomer-dimer entropy equal to $\max _{p \in[0,1]} h_{3}(p)$, given in [11]. h3Low is a lower bound implied by the maximal value of FT lower bound. ALMC is the function $g h_{6}(p)$ of $(5.2)$, conjectured to be a lower bound in the Asymptotic Lower Matching Conjecture. AUMC is the monomer-dimer entropy $h_{K}(p)$ of dimer density $p$ in a countable union of $K_{6,6}$, given by (6.3)-(6.5) and conjectured to be an upper bound by the Asymptotic Upper Matching Conjecture. Notice that AUMC goes a little over h3High: a countable union of $K_{6,6}$ has a higher monomer-dimer entropy than an infinite cubic grid.

\section{References}

[1] R.J. Baxter, Dimers on a rectangular lattice, J. Math. Phys. 9 (1968), 650-654.

[2] L.M. Bregman, Some properties of nonnegative matrices and their permanents, Soviet Math. Dokl. 14 (1973), 945-949.

[3] G.P. Egorichev, Proof of the van der Waerden conjecture for permanents, Siberian Math. J. 22 (1981), 854-859.

[4] P. Erdös and A. Rényi, On random matrices, II, Studia Math. Hungar. 3 (1968), 459464 . 


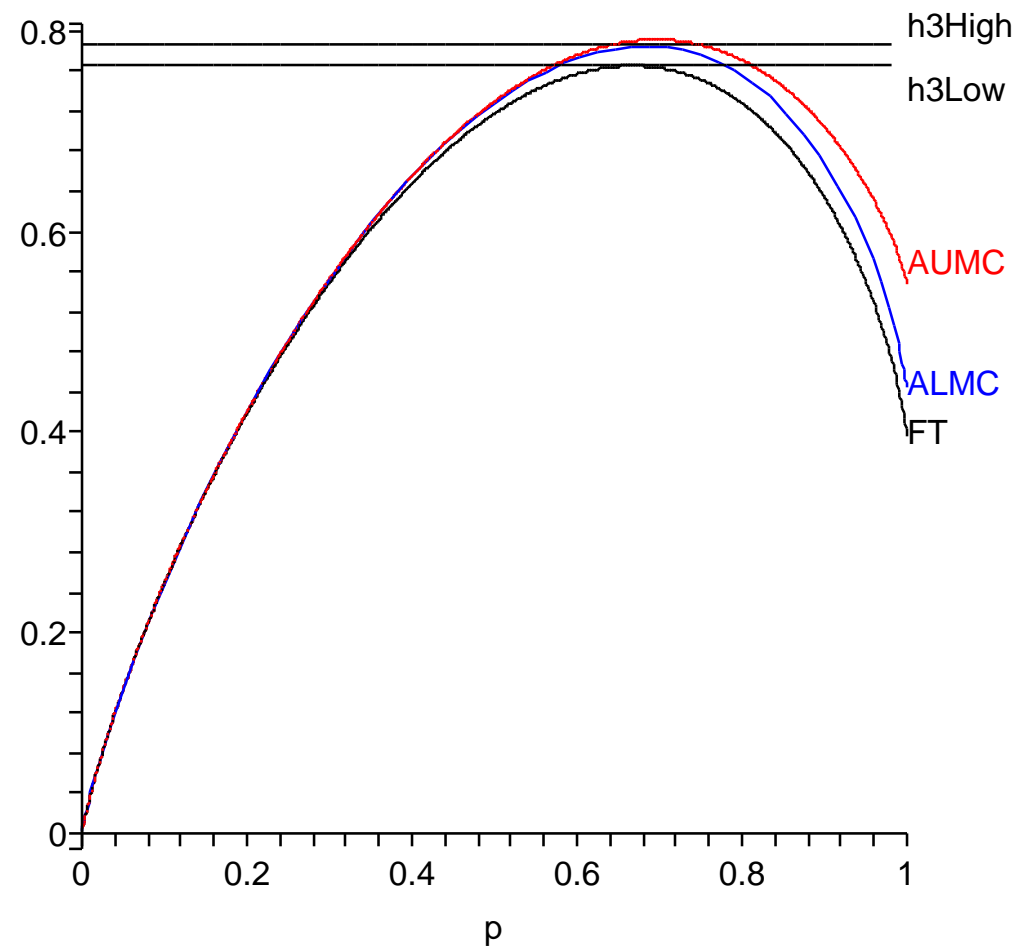

Figure 2: Monomer-dimer tiling of the 3-dimensional grid: entropy as a function of dimer density. FT is the Friedland-Tverberg lower bound, h3Low and h3High are the known bounds for the monomer-dimer entropy. ALMC is the Asymptotic Lower Matching Conjecture. AUMC is the entropy of a countable union of $K_{6,6}$, conjectured to be an upper bound by the Asymptotic Upper Matching Conjecture.

[5] D.I. Falikman, Proof of the van der Waerden conjecture regarding the permanent of doubly stochastic matrix, Math. Notes Acad. Sci. USSR 29 (1981), 475-479.

[6] S. Friedland, A lower bound for the permanent of doubly stochastic matrices, Ann. of Math. 110 (1979), 167-176.

[7] S. Friedland, A proof of a generalized van der Waerden conjecture on permanents, Lin. Multilin. Algebra 11 (1982), 107-120.

[8] S. Friedland and L. Gurvits, Generalized Friedland-Tverberg inequality: applications and extensions, arXiv:math/0603410 v2, 24 August, 2006.

[9] S. Friedland, E. Krop, P.H. Lundow and K. Markström, Computational Validations of the Asymptotic Matching Conjectures, arXiv:math.CO/0603001 v1, 28 February, 2006.

[10] S. Friedland, E. Krop and K. Markström, Averages for partial matchings in $r$-regular bipartite graphs, asymptotic conjectures and their verifications for $r=2$.

[11] S. Friedland and U.N. Peled, Theory of Computation of Multidimensional Entropy with an Application to the Monomer-Dimer Problem, Advances of Applied Math. 34(2005), 486-522.

[12] S. Friedland and U.N. Peled, The pressure associated with multidimensional SOFT, in preparation. 
[13] L. Gurvits , Combinatorial and algorithmic aspects of hyperbolic polynomials , arXIv: math.CO/0404474, 2004 .

[14] L. Gurvits, Hyperbolic polynomials approach to van der Waerden/Schrijver-Valiant like conjectures: Sharper bounds, simpler STOC'06: Proceedings of the 38th Annual ACM Symposium on Theory of Computing, 417-426, ACM, New York, 2006. arXIv:math.CO/0510452, 21 October, 2005.

[15] J.M. Hammersley, Existence theorems and Monte Carlo methods for the monomerdimer problem, in Reseach papers in statistics: Festschrift for J. Neyman, edited by F.N. David, Wiley, London, 1966, 125-146.

[16] O.J. Heilmann and E.H. Lieb, Theory of monomer-dimer systems., Comm. Math. Phys. 25 (1972), 190-232.

[17] L. Lovász and M.D. Plummer, Matching Theory, North-Holland Mathematical Studies, vol. 121, North-Holland, Amsterdam, 1986.

[18] A. Schrijver, Counting 1-factors in regular bipartite graphs, J. Comb. Theory B 72 (1998), 122-135.

[19] A. Schrijver and W.G.Valiant, On lower bounds for permanents, Indagationes Mathematicae 42 (1980), 425-427.

[20] R. Sinkhorn, A relationship between arbitrary positive matrices and doubly stochastic matrices, Ann. Math. Statist. 35 (1964), 876-879.

[21] H. Tverberg, On the permanent of bistochastic matrix, Math. Scand. 12 (1963), 25-35.

[22] B.L. van der Waerden, Aufgabe 45, Jber Deutsch. Math.-Vrein. 35 (1926), 117.

[23] I.M. Wanless, A lower bound on the maximum permanent in $\Lambda_{n}^{k}$, Linear Algebra Appl. 373 (2003), 153-167. 